LEISTUNGSENTGELT

\title{
Netzwerke erleichtern den Einstieg
}

Eckhard Eyer

Manche sozialen Dienste und Einrichtungen tun sich schwer mit der Einführung von Leistungsentgeltsystemen. In einem Netzwerk mit anderen Trägern kann dies besser, schneller, mit weniger Aufwand und geringerem Widerstand gelingen.

Im September 2005 wurde der Tarifvertrag des öffentlichen Dienstes (TVöD) unterzeichnet, der den Bundesangestelltentarif ablöst (BAT). Der neue Tarifvertrag des öffentlichen Dienstes sieht ab dem 1. Januar 2007 ein Leistungsentgelt in Form einer Leistungszulage oder Leistungsprämie in Höhe von zunächst durchschnittlich einem Prozent des Grundentgeltes vor. Das Leistungsentgelt soll im Laufe der Jahre auf acht Prozent an- ferenziert, also »mit der Gießkanne« auszahlen.

Das Leistungsentgelt soll nach dem Willen der Tarifvertragsparteien den Leistungsgedanken, der in der »freien Wirtschaft « weitgehend üblich ist, auch in den öffentlichen Dienst und die Sozialwirtschaft einführen. Das neue Leistungsentgelt soll motivierend sein und den Mitarbeitern einen Leistungsanreiz bieten. Die Gestaltungsmöglichkeiten, die der $\S 18$ des TVöD (vgl. Kasten) gibt, sind groß, um es den Verwaltungen und Einrichtungen zu erlauben, das betrieblich optimale Leistungsentgeltsystem zu gestalten. Hierzu ist von einer »betrieblichen Kommission «, die paritätisch von Vertretern des Managements und des Betriebsrates besetzt ist, das betriebliche Leistungsentgelt-

\section{»Hilfreicher Verweis bei Problemen: >Die Anderen machen es auch «"}

wachsen, was durchschnittlich einem Monatsgehalt an Leistungsentgelt im Jahr entspricht.

Die Einrichtungen des öffentlichen Dienstes hatten - seit Abschluss des Tarifvertrages - die Chance, mit 15 Monaten Vorlaufzeit ihre Dienstvereinbarungen zu erarbeiten, Führungskräfte und Mitarbeiter zu qualifizieren um so zum Jahresanfang in die neue Zeit zu starten. Die Einrichtungen, die bis zum 31. Juli 2007 keine Dienstvereinbarung abgeschlossen haben, müssen das tarifliche Leistungsentgelt für das Jahr 2007 undif- system zu entwickeln, das auf die betriebliche Situation, gegebenenfalls differenziert nach einzelnen Fachbereichen, zuzuschneiden ist.

\section{Herausforderung für die betriebli- che Kommission}

Die Gestaltung eines Leistungsentgeltsystems ist für Management- und Personal- oder Betriebsratsmitglieder, die in der betrieblichen Kommission mitarbeiten, eine große Herausforderung:

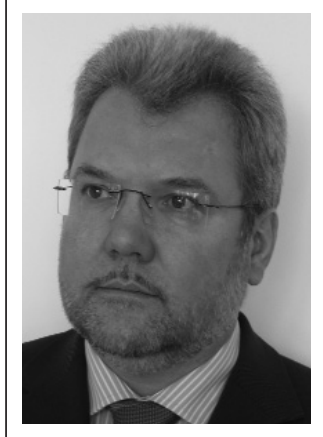

Eckhard

Eyer ist

Diplomin-

genieur,

Diplom-

kaufmann

und Vergütungsberater. $\mathrm{Er}$ ist ge-

schäftsführender Gesellschafter der Perspektive Eyer Consulting in Köln. Eyer beriet das erste "Modellprojekt Leistungsentgelt « der Caritas, er ist Berater der »Projektgesellschaft innovatives Arbeiten in caritativen Unternehmen " und hat bei verschiedenen Wohlfahrtsverbänden in der ambulanten und stationären Altenpflege sowie der Jugend- und Behindertenarbeit, Leistungs- und Erfolgsvergütung eingeführt. Zunehmend unterstützt er Unternehmen der Sozialwirtschaft die betriebliche Entgeltsysteme statt des TVöD einführen. Er ist Herausgeber des Buches »Entgeltsysteme für Dienstleister « und Autor des Buches "Leistungsentgelt nach TVöD erfolgreich einführen «. Zudem ist er Lehrbeauftragter an der Universität Trier.

Internet http://www.eyer.de

- Ein Leistungsentgeltsystem ist Neuland für den öffentlichen Dienst und die Sozialwirtschaft, Erfahrungen in der Non-Profit-Unternehmen fehlen bisher.

- Empfehlungen des Verbandes der kommunalen Arbeitgeberverbände (VKA) und der Vereinten Dienstleistungsgewerkschaft (ver.di) sind nicht aufeinander abgestimmt, sie widersprechen sich zum Teil und bieten so nur bedingt Orientierung.

- Vorbehalte der Führungskräfte und Mitarbeiter gegenüber dem neuen sensiblen Thema Leistungsentgelt sind groß, die Begriffe »Nasenfak- 


\section{$\S 18$ Leistungsentgelt}

\author{
$[\ldots]$
}

(5) Die Feststellung oder Bewertung von Leistungen geschieht durch das Vergleichen von Zielerreichungen mit den in der Zielvereinbarung angestrebten Zielen oder über eine systematische Leistungsbewertung. Zielvereinbarung ist eine freiwillige Abrede zwischen der Führungskraft und einzelnen Beschäftigten oder Beschäftigtengruppen über objektivierbare Leistungsziele und die Bedingungen ihrer Erfüllung. Leistungsbewertung ist die auf einem betrieblich vereinbarten System beruhende Feststellung der erbrachten Leistung nach möglichst messbaren oder anderweitig objektivierbaren Kriterien oder durch aufgabenbezogene Bewertung.

(6) Das jeweilige System der leistungsbezogenen Bezahlung wird betrieblich vereinbart. Die individuellen Leistungsziele von Beschäftigten bzw. Beschäftigtengruppen müssen beeinflussbar und in der regelmäßigen Arbeitszeit erreichbar sein. Die Ausgestaltung geschieht durch Betriebsvereinbarung oder einvernehmliche Dienstvereinbarung, in der insbesondere geregelt werden:

- Verfahren der Einführung von leistungs- und/oder erfolgsorientierten Entgelten,

- zulässige Kriterien für Zielvereinbarungen,

- Ziele zur Sicherung und Verbesserung der Effektivität und Effizienz, insbesondere für Mehrwertsteigerungen (z. B. Verbesserung der Wirtschaftlichkeit, der Dienstleistungsqualität, der Kunden-/Bürgerorientierung)

- Auswahl der Formen von Leistungsentgelten, der Methoden sowie Kriterien der systematischen Leistungsbewertung und der aufgabenbezogenen Bewertung (messbar, zahlbar oder anderweitig objektivierbar), ggf. differenziert nach Arbeitsbereichen, u. U. Zielerreichungsgrade,

- Anpassung von Zielvereinbarungen bei wesentlichen Änderungen von Geschäftsgrundlagen,

- Vereinbarung von Verteilungsgrundsätzen,

- Überprüfung und Verteilung des zur Verfügung stehenden Finanzvolumens, ggf. Begrenzung individueller Leistungsentgelte aus umgewidmetem Entgelt,

- Dokumentation und Umgang mit Auswertungen über Leistungsbewertungen.

(7) Bei der Entwicklung und beim ständigen Controlling des betrieblichen Systems wirkt eine betriebliche Kommission mit, deren Mitglieder je zur Hälfte vom Arbeitgeber und vom Betriebs-/Personalrat aus dem Betrieb benannt werden. Die betriebliche Kommission ist auch für die Beratung von schriftlich begründeten Beschwerden zuständig, die sich auf Mangel des Systems bzw. seiner Anwendung beziehen. Der Arbeitgeber entscheidet auf Vorschlag der betrieblichen Kommission, ob und in welchem Umfang der Beschwerde im Einzelfall abgeholfen wird. Folgt der Arbeitgeber dem Vorschlag nicht, hat er seine Gründe darzulegen. Notwendige Korrekturen des Systems bzw. von Systembestandteilen empfiehlt die betriebliche Kommission. Die Rechte der betrieblichen Mitbestimmung bleiben unberührt.

Aus dem Tarifvertrag für den öffentlichen Dienst (TVöD) vom 13. September 2005 tor « und »Subjektivität« tauchen in den Mitarbeiterversammlungen auf.

- Eine belastbare Datenbasis für Zielvereinbarungen fehlt in den meisten Fällen.

- Es gibt keine vom Verband der kommunalen Arbeitgeberverbände und von ver.di gemeinsam empfohlenen Modellbeispiele, so genannte »Leuchttürme «, die den betrieblichen Kommissionen Orientierung geben.

In dieser Situation stehen die Verwaltungen und Einrichtungen vor der Frage, ob sie den Einführungsprozess als einzelne Einrichtung bestreiten oder im Geleitzug mit befreundeten Einrichtungen und Unternehmen, die in einer ähnlichen Situation aber keine direkten Konkurrenten sind.

\section{Netzwerke bilden}

In der beschriebenen Situation bietet es sich an Synergien zu nutzen; beispielsweise innerhalb einer Teilbranche der Sozialwirtschaft, die ähnlich strukturiert ist, überbetrieblich zusammenzuarbeiten. So geschehen im Jahre 2006, als mehrere Werkstätten für behinderte Menschen (WfbM) sich in einer Region zusammentaten. Das war in diesem Arbeitsfeld gut möglich, weil die Einzugsbereiche definiert sind und keine Konkurrenz um Kunden besteht.

Zunächst setzten sich die Einrichtungsleiter in einem eintägigen Seminar mit dem Leistungsentgelt nach $\S$ 18 TVöD auseinander. Sie erörterten neben den Gestaltungsoptionen und dem betrieblichen Projektmanagement das Für und Wider einer überbetrieblichen Zusammenarbeit und entschieden sich dann für eine überbetriebliche Zusammenarbeit kombiniert mit der parallelen betrieblichen Arbeit in ihren betrieblichen Kommissionen, die in der Regel aus sechs Vertretern aus jedem Betrieb bestanden.

\section{Balance zwischen Netzwerk und Einrichtung}

Die Balance der Arbeit im überbetrieblichen Netzwerk und der in der Einrichtung ist wichtig, weil zum einen die Synergien genutzt werden 


\section{Aufbau einer Projektstruktur}

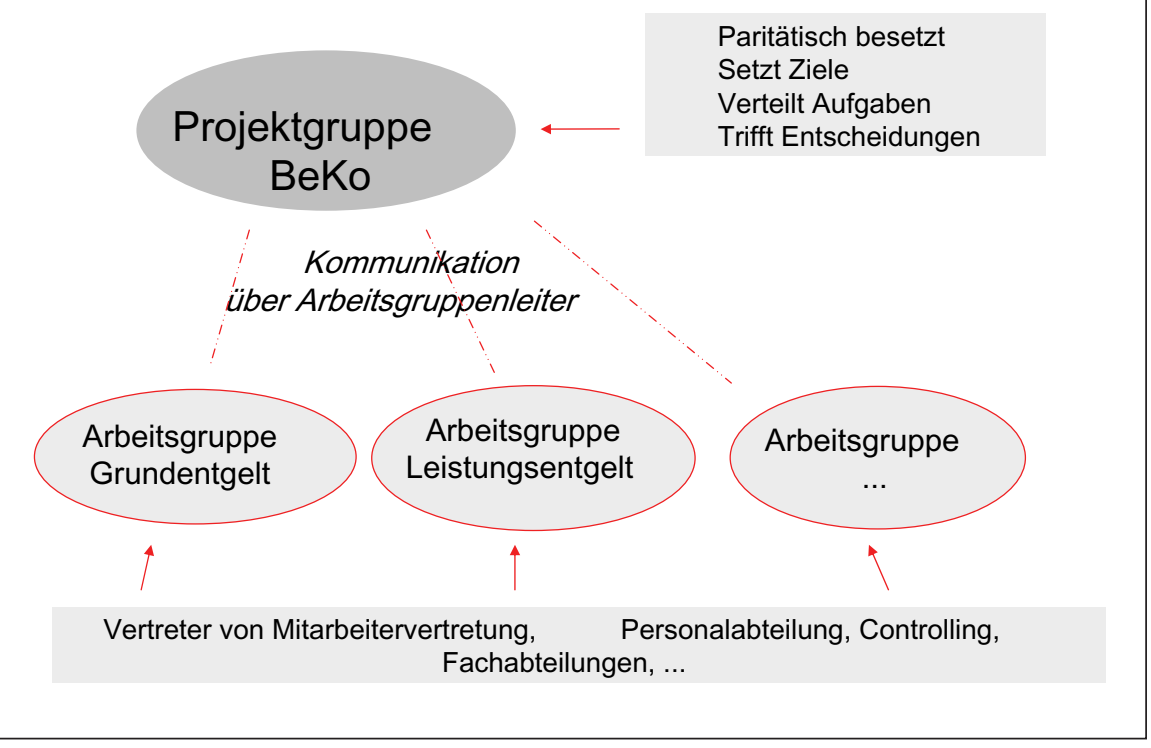

und zum anderen die Führungskräfte und Mitarbeiter angemessen an dem Prozess beteiligt werden müssen. Ein überbetrieblich erarbeitetes Leistungsentgeltsystem darf den Mitarbeitern und Führungskräften nicht einfach übergestülpt werden, wenn es erfolgreich gelebt werden soll.

Die Verknüpfung der betrieblichen mit der überbetrieblichen Ebene ist auch notwendig, weil der überbetriebliche Gestaltungs- und Lernprozess immer wieder betrieblich geerdet werden muss. Dies geschieht beispielsweise, indem man betriebliche Ziele für Zielvereinbarungen entwickelt, sie an dem eigenen Controlling und Qualitätsmanagement orientiert und sich darüber und über die gesammelten Erfahrungen überbetrieblich austauscht und von dort Impulse bekommt. Der Prozess ist von einem erfahrenen Vergütungsexperten zu moderieren, der auch einen fachlichen Input zum Leistungsentgelt geben kann. Die Erfahrung

\section{Überbetriebliche Kooperationen}

\section{Beispiel Werkstätten für behinderte Menschen 2006/2007}
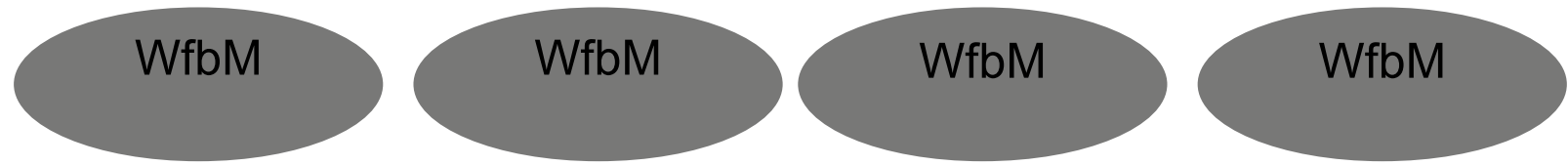

Gemeinsame Information über die Gestaltung eines Leistungsentgeltsystem

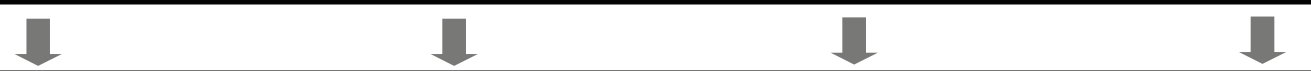

betriebl. Entscheidung: Leistungsentgelt satt leistungsunabhängiger Auszahlung

Netzwerk zieht Berater hinzu; gemeinsame Schulung der Betrieblichen Kommissionen, Vorgehen im Netzwerk und betrieblich abstimmen

I $\mathbb{V}$

Erarbeitung der einrichtungsspezifischenLeistungsentgeltsysteme (LE-Systeme)

Vorstellung und Diskussion der einrichtungsspezifischen LE-Systeme, Benchmarking

einrichtungsspezifische Information und Schulung der Mitarbeiter und Führungskräfte

einrichtungsspezifischer Trockenlauf und anschließende Umsetzung mit finanziellen

Auswirkungen 
in einem Verbund von Großstädten in Nordrhein-Westfalen, die in der Aus- und Weiterbildung zusammenarbeiten und 2006 gemeinsam im Netzwerk das Leistungsentgelt zum TVöD vorbereiten wollten, zeigt, dass die Beratung durch einen Arbeitsrechtler als Moderator dem überbetrieblichen Prozess nicht weiterhilft, weil zunächst nicht rechtliche Fragen, sondern inhaltliche Gestaltungsoptionen und das Projektmanagement bei der Einführung, die angemessene Beteiligung der Führungskräfte und Mitarbeiter im Vordergrund stehen.

\section{Erfolgsfaktoren für Netzwerke}

Als Faktor für die erfolgreiche $\mathrm{Zu}$ sammenarbeit in Netzwerken ist zunächst zu nennen: Nur Einrichtungen sollten sich zusammenschließen, die nicht direkte Konkurrenten sind. Sie sollten regional und fachlich gut zusammenpassen und die gleichen Einführungszeitpunkte und Einführungszeiträume planen. Dies ist notwendig, damit alle Einrichtungen das gleiche inhaltliche und zeitliche Ziel haben und mit gleicher Intensität am Thema arbeiten. Will eine Einrichtung nur von anderen profitieren, ohne sich selbst zu engagieren, so zeigt die Erfahrung in anderen Branchen, kommt es zu Konflikten zwischen den beteiligten Unternehmen und gegebenenfalls sogar zum Ausschluss eines Unternehmens, das sich nicht einbringt.

Die Unternehmen, die den betrieblichen Prozess nicht konsequent betreiben, können nur bedingt davon profitieren. Sie können nicht wirklich für den Prozess lernen, weil sie die Erfahrungen nicht teilen können. Der fachliche Input muss von Experten in Sachen Gestaltung und Einführungsmanagement von Entgeltsystemen moderiert - besser geführt - werden. Die Gestaltung der Betriebsvereinbarungen ist »nur « die Dokumentation des einrichtungsspezifisch erarbeiteten Leistungsentgeltsystems, die sich an Musterbetriebsvereinbarungen orientiert.

\section{Erfahrungen}

Die Erfahrung mit mehreren Netzwerken zeigt, dass die erarbeiteten
Leistungsvergütungssysteme den gleichen Geist in sich tragen, dass die Ausgestaltung aber in Abhängigkeit vom Leitbild und insbesondere in Abhängigkeit von der Datenbasis und deren Qualität sowie der Unternehmens- und Leistungskultur variiert:

- Einrichtungen, die ein stark ausgefeiltes Controlling haben, im Rahmen ihres Qualitätsmanagement über Kennzahlen und Audits verfügen neigen eher zu Zielvereinbarungen.

- Einrichtungen, die über diese Daten nicht verfügen, beginnen oft mit der systematischen Leistungsbewertung und Leistungsbeurteilung und haben vielleicht mittelfristig das Ziel, die Datenbasis für Zielvereinbarungen zu schaffen.

- Unternehmen, die im Rahmen ihres Qualitätsmanagements Mitarbeiterbeurteilungsgespräche durchführen oder gar Zielvereinbarungen ohne materielle Auswirkungen seit Jahren eingeführt haben, können auf einer sehr guten Basis aufbauen und zügig Zielvereinbarungen mit Leistungsprämie einführen.

Die Schulungen in Leistungsbewertung und Zielvereinbarungen wurden in dem vorgenannten Netzwerk - zugeschnitten auf die betrieblichen Leistungsentgeltsysteme - einrichtungsspezifisch durchgeführt. Sie basierten auf dem jeweils betrieblichen Verfahren und berücksichtigten die Vorkenntnisse und die betriebliche Übung bei Mitarbeitergesprächen, Personalentwicklungsgesprächen und Zielvereinbarungen ohne finanzielle Auswirkungen.

\section{Bewertung der Teilnehmer}

Am höchsten schätzten sowohl Vertreter des Managements als auch des Betriebsrats bei einer Befragung der betrieblichen Kommissionen die Kommunikation unter Kollegen im Netzwerk, die nicht separiert nach Arbeitgebern und Arbeitnehmern verlief. Inhaltlich war das Gespräch über gemeinsame Probleme bei dem Gestaltungs- und vor allem Kommunikationsprozess in den Einrichtungen wichtig. Das Gefühl, nicht allein auf weiter Flur zu sein, war ebenso wichtig wie das Argument, wenn es in der eige- nen Einrichtung einmal schwierig wurde: »Die Anderen machen es auch.«

Das in der Planungsphase wichtigere Thema »Synergien « insbesondere bei den Kosten (Warum das Rad zwei Mal erfinden?) trat demgegenüber bei der Befragung überraschenderweise zurück. Mittlerweile gibt es auch vereinzelt Netzwerke zur gemeinsamen Gestaltung von neuen betrieblichen Grund- und Leistungsentgeltsystemen. Die Teilnehmer dieser Netzwerke wollen den BAT ablösen, aber den TVöD, den sie für verbesserungswürdig und nicht finanzierbar halten, vermeiden.

\section{Fazit}

Einrichtungen der Sozialwirtschaft schließen sich zunehmend mit ähnlichen oder gleich gelagerten Einrichtungen zusammen und erarbeiten in einer intelligenten Arbeitsteilung zwischen überbetrieblicher Zusammenarbeit der betrieblichen Kommissionen und der Arbeit in den Einrichtungen erfolgreich neue Leistungsentgeltsysteme.

Der Erfahrungsaustausch und das gemeinsame Arbeiten an einem für die Branche neuen Thema werden als wertvoll erlebt, ebenso die Synergien und die kompetente Moderation mit fachlichem Input der überbetrieblichen Zusammenkünfte.

Die Erfahrungen bei der Gestaltung von Leistungsentgeltsystemen lassen sich auch auf die Gestaltung betrieblicher Grund- und Leistungsentgeltsysteme übertragen. 